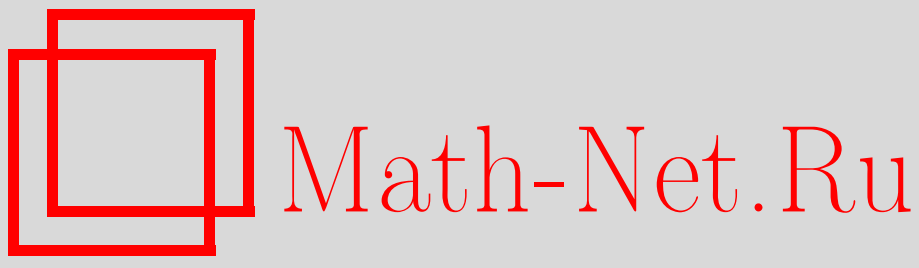

Д. П. Желобенко, Дифференциальные операторы в градуированных алгебрах, Изв. РАН. Сер. матем., 1996, том 60, выпуск 2, 49-72

DOI: https://doi.org/10.4213/im71

Использование Общероссийского математического портала Math-Net.Ru подразумевает, что вы прочитали и согласны с пользовательским соглашением

http://www . mathnet.ru/rus/agreement

Параметры загрузки:

IP: 3.93 .64 .190

26 апреля 2023 г., 14:03:44 
УДК 519.46

\author{
Д. П. Желобенко
}

\title{
Дифференциальные операторы в градуированных алгебрах
}

\begin{abstract}
В статье рассматриваются общие вопросы, связанные с определением и исследованием абстрактных дифференциальных операторов в ассоциативных градуированных алгебрах. Исследуются связи между специальными классами таких операторов. Доказываются некоторые структурные теоремы и некоторые аналоги теоремы плотности для алгебры дифференциальных операторов. Рассматриваются в качестве иллюстрации классические и квантовые алгебры Серра.

Библиографиия: 14 наименований.
\end{abstract}

Понятие дифференщиального оператора на алгебраическом многообразии относится к числу основных понятий алгебраической геометрии. Дифференциальные операторы на многообразии $M$ суть линейные операторы, действуюшие в алгебре $A(M)$ регулярных функций на $M$ и связанные некоторыми соотношениями нильпотентности с операторами умножения на функции из $A(M)$. При этом существенна коммутативность структурной алгебры $A(M)$.

Изложенная конструкция допускает двойное обобшение, в духе обшей программы развития "некоммутативной алгебраической геометрии", намеченной, например, во введении к книге [2]. С одной стороны, речь по-прежнему идет об исследовании линейных операторов, действуюших в некоторой алгебре $A$, причем эта алгебра предполагается ассоциативной, но не обязательно коммутативной. Отказ от коммутативности в известной степени компенсируется другими ограничениями, которые в нашем контексте сводятся к наличию некоторой специальной градуировки относительно адлитивной упорядоченной группы (например, группы $\mathbb{Z}$ ). $\mathrm{C}$ другой стороны, как подсказывает недавнее развитие теории квантовых групп, в ситуации, связанной с градуированными алгебрами, естественно рассматривать некоторые "подкрученные" дифференциальные операторы, определение которых основано на надлежащем обобщении понятия коммутатора в градуированных алгебрах. Впрочем, этот подход широко используется в частном случае $\mathbb{Z}_{2}$-градуированных алгебр (супералгебр), в том числе в их известных приложениях к задачам алгебраической топологии (см., например, [13]).

Содержание статьи сводится к следующему. В $\S 1$ изложены предварительные сведения и основные определения. Дается определение алгебры дифференциальных операторов $D(A)$, ассоциированной с градуированной алгеброй $A$. Алгебра $D(A)$ порождается своей подалгеброй $A$ и некоторой дуальной подалгеброй $D$, причем выполняются следуюшие соотношения: $D(A)=A D=D A$. В $\S 2$ изложены вспомогательные результаты, связанные с рассмотрением некоторых билинейных форм в алгебре $D(A)$. Здесь же вводятся три специальных класса алгебр $A$

Работа выполнена при финансовой поддержке Российского Фонда фундаментальных исследований (грант 95-01-0233).

(C) Д.П. Желовенко 1996 
(регулярные, вполне дифференцируемые, невырожденные), основные результаты для которых собраны в $\S 3$. Остановимся на кратком обзоре этих результатов.

(А) Регулярные алгебры $A$ (см. п. 2.1) характеризуются наличием двойственности между векторными пространствами $A, D$, что сводится к наличию достаточно большого запаса дифференциальных операторов в алгебре $A$. Сушественный смысл этого понятия раскрывается в теореме 3.1 , основное содержание которой представляет собой градуированный аналог теоремы плотности для алгебры $D(A)$. В частности, алгебра $D(A)$ оказывается плотной в $\operatorname{End} A$ (относительно поточечной сходимости).

(В) Вполне дифферениируемые алгебры $A$ (см. п. 2.3) обладают достаточно большим запасом операторов первого порядка. Для этих алгебр сушествуют простые критерии регулярности и невырожденности (теорема 3.4).

(C) Невырожденные алгебры $A$ (см. п. 2.8) допускают полное описание в терминах образующих и соотношений между ними. В этом случае алгебра $D(A)$ порождается операторами первого порядка. Более того, в этом случае двойственность между алгебрами $A, D$ становится симметричной (инволютивной), так что алгебры $A, D$ сопряжены друг другу относительно некоторой инволюции алгебры $D(A)$ (теорема 3.6).

В $\S 4$ приводится иллюстрация этих результатов на примере классических и квантовых алгебр Серра. Следует отметить, что алгебры Серра представляют собой естественный объект некоммутативной алгебраической геометрии. С одной стороны, эти алгебры в известном смысле близки к коммутативным. С другой стороны, хорошо известна роль этих алгебр в структурной теории и теории представлений других алгебраических структур (редуктивные алгебры Ли, алгебры Каца-Муди, алгебры Дринфельда-Джимбо). Например, каждая алгебра Дринфельда-Джимбо может быть реализована (см. п. 4.9) посредством дифференциальных операторов, действующих в борелевской оболочке соответствующей квантовой алгебры Серра.

Интересно отметить, что классические алгебры Серра $X(a)$ вырождаются в смысле п. 2.8 (см. п. 3.10), в то время как их квантовые аналоги $X_{q}(a)$ невырождены (см. п. 4.6). Впрочем, это соответствует известному принципу квантовой теории, согласно которому "квантование снимает вырождение".

В $\S 5$ описаны все невырожденные алгебры в терминах образуюших и структурных коэффищиентов (предложение 5.5).

Наконец, в $\S 6$ определяются некоторые аналоги псевдодифференциальных операторов в алгебре $A$. Показано (теорема 6.4), что в невырожденном случае алгебра псевдодифференциальных операторов достаточно велика, что также может рассматриваться как некоторый вариант теоремы плотности в терминах формальных рядов над алгеброй $D(A)$.

Изложение в этой статье носит в основном элементарный характер. Исключение составляет лишь $\S 4$, в котором используются несколько более специальные сведения, относящиеся к алгебрам Серра. Более детальное исследование алгебр $D(A)$ предполагается изложить в отдельной статье.

Элементы алгебры $D(A)$ допускают естественную “квантовую” трактовку в терминах (обобщенных) операторов рождения и уничтожения. В этом смысле построение алгебры $D(A)$ можно рассматривать как восстановление полной алгебры наблюдаемых по ее подалгебре, образующие которой интерпретируются как операторы рождения. При этом существенно, что $D(A)$ действует в $A$ (обобщенное 
представление Фока).

Содержание этой статьи может также рассматриваться в рамках общей теории контрагредиентных алгебр (см., например, [6], [7]). Во всяком случае, если алгебра $A$ невырождена, то $D(A)$ есть невырожденная контрагредиентная алгебра в смысле [7] и пространство $A$ есть универсальный модуль Верма над алгеброй $D(A)$.

\section{§1. Основные определения}

1.1. Пусть $A$ - ассоциативная алгебра с единицей над полем $F$, градуированная подпространствами $A_{\alpha}, \alpha \in \Gamma$, где $\Gamma$ - некоторая аддитивная группа. Иначе говоря, имеем

$$
A=\bigoplus_{\alpha \in \Gamma} A_{\alpha}, \quad A_{\alpha} A_{\beta} \subset A_{\alpha+\beta}
$$

для всех $\alpha, \beta \in \Gamma$.

Элементы $x \in A_{\alpha}$ называются однородными степени $\alpha$, с обозначением $\operatorname{deg} x=\alpha$ (так что символ $\operatorname{deg} x$ однозначно определен при $\left.0 \neq x \in A_{\alpha}\right)$. Подпространство $B \subset A$ называется градуированным (или Г-градуированны.м), если оно совпадает с суммой своих однородных компонент $B_{\alpha}=B \cap A_{\alpha}$.

Заметим также, что $A_{0}$ есть подалгебра с единищей алгебры $A$ (здесь 0 - нейтральный элемент группы $\Gamma$ ).

1.2. Элемент $a \in$ End $A$ называется однородны.м степени $\alpha$, если $a A_{\beta} \subset A_{\alpha+\beta}$ для всех $\beta \in \Gamma$. Однородные элементы степени $\alpha$ образуют подпространство $E(A)_{\alpha} \subset \operatorname{End} A$. Легко видеть, что эти подпространства линейно независимы. Их прямая сумма

$$
E(A)=\bigoplus_{\alpha \in \Gamma} E(A)_{\alpha}
$$

есть Г-градуированная алгебра (подалгебра в End $A$ ). Элементы $a \in E(A)$ называются градуированными (или Г-градуированными) операторами в алгебре $A$.

$\mathrm{B}$ частности, для каждого $a \in A$ оператор левого умножения $l(a) x=a x$, где $x \in A$, градуирован. Отображение $a \mapsto l(a)$ инъективно (поскольку $A$ содержит единицу) и определяет вложение градуированных алгебр $A \rightarrow E(A)$.

В дальнейшем мы отождествляем элементы $a, l(a)$, т.е. рассматриваем $A$ как подалгебру в $E(A)$.

1.3. Числовая функция $\varepsilon: \Gamma \times \Gamma \rightarrow F$ называется мультипликатором (или бихарактером) группы $\Gamma$, если каждая из функций $\varepsilon(\alpha, \cdot), \varepsilon(\cdot, \beta)$ есть характер группы $\Gamma$. В частности, $\varepsilon(\alpha, 0)=\varepsilon(0, \beta)=1$ для всех $\alpha, \beta \in \Gamma$. Полагая

$$
\varepsilon(x, y)=\varepsilon(\alpha, \beta) \quad \text { при } \quad \operatorname{deg} x=\alpha, \quad \operatorname{deg} y=\beta,
$$

получаем новую функцию $\varepsilon$, определенную на декартовых произведениях $A_{\alpha} \times A_{\beta}$ и мультипликативную по каждому аргументу, т.е.

$$
\varepsilon(x y, z)=\varepsilon(x, z) \varepsilon(y, z), \quad \varepsilon(x, y z)=\varepsilon(x, y) \varepsilon(x, z)
$$

для всех однородных $x, y, z \in A$. Помимо этого имеем $\varepsilon(x, z)=\varepsilon(z, y)=1$ для всех однородных $x, y \in A$ и всех $z \in A_{0}$. Выражение

$$
[x, y]_{\varepsilon}=x y-\varepsilon(x, y) y x
$$


билинейно на каждом декартовом произведении $A_{\alpha} \times A_{\beta}$ и потому продолжается по билинейности до операции умножения, называемой $\varepsilon$-коммутатором элементов $x, y \in A$.

Используя соотношения мультипликативности (1.2), находим

$$
\begin{aligned}
& {[x y, z]_{\varepsilon}=x[y, z]_{\varepsilon}+\varepsilon(y, z)[x, z]_{\varepsilon} y,} \\
& {[x, y z]_{\varepsilon}=[x, y]_{\varepsilon} z+\varepsilon(x, y) y[x, z]_{\varepsilon}}
\end{aligned}
$$

для всех однородных $x, y, z \in A$.

Числовая функция (1.1) назьвается структурным коэффициентом алгеб$p$ b $A$. Мы будем иногда использовать обозначения $(A, \varepsilon), E(A, \varepsilon)$ вместо $A, E(A)$ (соответственно) в том случае, когда в данной алгебре фиксирован структурный коэффициент (1.1).

1.4. Однородный элемент $a \in \operatorname{End} A$ называется дифференцированием (или $\varepsilon$-дифференцированием) алгебры $A$, если

$$
a(x y)=a(x) y+\varepsilon(a, x) x a(y)
$$

для всех однородных $x, y \in A$. Здесь функция $\varepsilon$ рассматривается как структурный коэффициент алгебры $E(A, \varepsilon)$. Соотношение (1.6) остается справедливым для всех $y \in A$ и поэтому может быть записано в виде

$$
[a, x]_{\varepsilon}=a(x),
$$

где элементы $x, a(x)$ рассматриваются как операторы левого умножения в алгебpe $A$.

Полагая $x=y=1$ в равенстве (1.6), находим $a(1)=0$, при условии $\operatorname{char} F \neq 2$.

Заметим, что функция $\varepsilon=1$ (т.е. $\varepsilon(\alpha, \beta)=1$ для всех $\alpha, \beta \in \Gamma$ ) есть мультипликатор групшы $Г$. Соотношения (1.3), (1.6) в этом случае сводятся к обычным определениям коммутатора и дифференцирования в алгебре $A$.

1.5. Алгебра $A$ назьвается стандартной, если она обладает системой однородных образующих $x_{i}(i \in I)$, для которых элементы $\alpha_{i}=\operatorname{deg} x_{i}$ линейно независимы над $\mathbb{Z}_{+}$, т.е. равенство $\sum_{i} n_{i} \alpha_{i}=0$, где $n_{i} \in \mathbb{Z}_{+}$, возможно только при $n_{i}=0$ для всех $i \in I$. В этом (и только этом) случае отношение $\alpha \geqslant \beta$ при $\alpha-\beta \in \Gamma_{+}$, где $\Gamma_{+}=\sum_{i} \mathbb{Z}_{+} \alpha_{i}$, наделяет группу $\Gamma$ структурой упорядоченной группы, при которой $\alpha_{i}>0$ для всех $i \in I$.

Заменяя группу Г соответствующей подгруппой, будем считать, не ограничивая обшности, что Г порождается элементами $\alpha_{i}(i \in I)$.

Если алгебра $A$ стандартна, то $A_{\alpha}=0$ при $\alpha \notin \Gamma_{+}$(т.е. $A$ обладает $\Gamma_{+}$-градуировкой), $A_{0}=F \cdot 1$. Отсюда

$$
A=F \oplus \sum_{i} x_{i} A
$$

где $A_{0}=F \cdot 1$ отождествляется с полем $F$.

Очевидно, каждая $\Gamma_{+}$-градуированная алгебра $A$ обладает более грубой $\mathbb{Z}_{+}$-градуировкой $A_{n} \quad\left(n \in \mathbb{Z}_{+}\right)$, определяемой степенями $|\alpha|=\sum_{i} n_{i}$ при $\alpha=\sum_{i} n_{i} \alpha_{i}$. Иначе говоря, $A_{n}$ есть сумма компонент $A_{\alpha}$ при $|\alpha|=n$. 
Если алгебра $A$ стандартна, то будем считать, что элементы $x_{i}(i \in I)$ линейно независимы, т.е. образуют базис в $A_{1}$. Нетрудно видеть (из соображений градуировки), что $x_{i}(i \in I)$ есть минимальная система $\mathbb{Z}$-однородных образующих алгебры $A$. Более того, эта система единственна с точностью до автоморфизма $\mathbb{Z}$-градуированной алгебры $A$ (поскольку $A_{1}$ есть наименьшее градуированное подпространство, порождающее алгебру $A$ ). То же верно в терминах Г-градуировки, если элементы $\alpha_{i}(i \in I)$ просты (т.е. их невозможно представить в виде $\alpha+\beta$, где $\left.0 \neq \alpha, \beta \in \Gamma_{+}\right)$.

Алгебра $A$ назьвается алгеброй конечного типа, если $\operatorname{dim} A_{\alpha}<\infty$ для всех $\alpha \in \Gamma$. Если $A$ - стандартная конечнопорожденная алгебра (card $I<\infty)$, то $A$ есть алгебра конечного типа даже в смысле $\mathbb{Z}_{+}$-градуировки, т.е. $\operatorname{dim} A_{n}<\infty$ для всех $n \in \mathbb{Z}_{+}$.

ПримеР. Пусть $A$ - свободная алгебра с образуюшими $x_{i}(i \in I)$, и пусть $\Gamma$ - аддитивная группа с образуюшими $\alpha_{i}(i \in I)$. В этом случае существует единственная Г-градуировка алгебры $A$, определяемая на образуюших по правилу $\operatorname{deg} x_{i}=\alpha_{i}(i \in I)$.

1.6. Пусть $(A, \varepsilon)$ есть стандартная алгебра. Определим подпространство $D \subset$ $E(A)$ как наибольшее подпространство, удовлетворяющее следующим условиям:

( $\alpha) \quad D_{-\alpha}=0$ при $\alpha \notin \Gamma_{+}$;

(ß) $D_{0}=F$;

$(\gamma) \quad\left[D, x_{i}\right]_{\varepsilon} \subset D$ для всех $i \in I$.

Из этого определения следует, что подпространство $D$ градуировано. Действительно, пусть $\bar{D}$ - прямая сумма компонент $D_{\alpha}(\alpha \in \Gamma)$. Нетрудно видеть, что $\bar{D}$ удовлетворяет условиям $(\alpha),(\beta),(\gamma)$, откуда $\bar{D}=D$. С другой стороны, пусть $\widetilde{D}-$ подалгебра алгебры $E(A)$, порожденная элементами $a \in D$. Применяя правило коммутирования (1.4) к одночленам от этих элементов, находим, что $\widetilde{D}$ удовлетворяет условиям $(\alpha),(\beta),(\gamma)$, откуда $\widetilde{D}=D$. Таким образом, $D$ есть градуированная подалгебра алгебры $E(A)$.

Согласно $(\beta),(\gamma)$ однородные компоненты $D_{-\alpha}\left(\alpha \in \Gamma_{+}\right)$определяются рекуррентно следующим образом: $D_{-\alpha}$ есть множество всех элементов $a \in E(A)_{-\alpha}$, удовлетворяющих соотношениям

$(\delta) \quad\left[a, x_{i}\right]_{\varepsilon} \in D_{-\alpha+\alpha_{i}} \quad$ для всех $i \in I$.

$\mathrm{C}$ другой стороны, имеем

(ع) $a\left(A_{\beta}\right)=0$ при $\beta<\alpha$

(поскольку это верно для всех элементов $a \in E(A)_{-\alpha}$ ).

Легко проверить, что соотношение $\operatorname{deg} a=-\alpha$ вытекает из условий $(\delta),(\varepsilon)$. Поэтому $D_{-\alpha}$ можно определить как множество всех элементов $a \in E(A)$, удовлетворяющих соотношениям $(\delta),(\varepsilon)$.

1.7. ПрЕДЛОЖЕНИЕ. Пусть А есть стандартная алгебра (см. $n .1 .5), u$ пусть $D(A)$ - подалгебра в $E(A)$, порожсденная подалгебрами $A, D$ (см. п. 1.6). Тогда имеем:

(i) коммутатор $\left[D_{-\alpha}, A_{\beta}\right]_{\varepsilon}, \alpha, \beta \in \Gamma_{+}$, содерәсится в подпространстве

$$
C_{\alpha \beta}=\sum A_{\gamma} D_{-\delta}=\sum D_{-\delta} A_{\gamma}
$$


где сумма берется по индексам $\gamma<\beta, \delta<\alpha$;

(ii) в алгебре $D(A)$ выполняются соотношения

$$
D(A)=A D=D A
$$

(iii) алгебра $D(A)$ обладает $\Gamma_{+}-$фильтрацией $D_{\alpha}(A)$, определяемой как сумма подпространств $A D_{-\beta}$ при $\beta \leqslant \alpha$.

Таким образом,

$$
D(A)=\bigcup_{\alpha \in \Gamma_{+}} D_{\alpha}(A), \quad D_{\alpha}(A) D_{\beta}(A) \subset D_{\alpha+\beta}(A)
$$

для всех $\alpha, \beta \in \Gamma_{+}$.

ДокАЗАТЕЛЬСТво. (i) Утверждение очевидно при $\beta=0$. В обшем случае индукция по $|\beta|$. Заметим вначале, что равенство двух сумм в определении (1.9) вытекает из допущения индукции по $|\beta|$. Для проведения дальнейшей индукции достаточно заменить $A_{\beta}$ на $x_{i} A_{\beta}$, где $i \in I$. Используя правило коммутирования (1.5), условие $(\gamma)$ и допушение индукции для индекса $\beta$, находим

$\left[D_{-\alpha}, x_{i} A_{\beta}\right]_{\varepsilon} \subset\left[D_{-\alpha}, x_{i}\right]_{\varepsilon} A_{\beta}+x_{i}\left[D_{-\alpha}, A_{\beta}\right]_{\varepsilon} \subset D_{-\alpha+\alpha_{i}} A_{\beta}+x_{i} C_{\alpha \beta} \subset C_{\alpha, \beta+\alpha_{i}}$,

откуда следует (i) для всех $\alpha, \beta \in \Gamma_{+}$.

(ii) Применяя правило (i), находим, что каждый одночлен от образующих, лежащих в $A \cup D$, содержится в $A D$ (аналогично в $D A$ ).

(iii) Применяя правило (i), находим $D_{-\beta} A \subset D_{\alpha}(A)$ для всех $\beta \leqslant \alpha$, откуда вытекают соотношения мультипликативности для подпространств $D_{\alpha}(A)$.

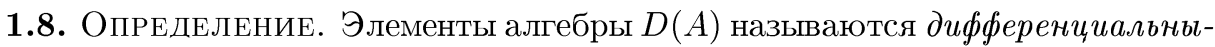
ми операторами в алгебре $A=(A, \varepsilon)$. Элементы $a \in D_{\alpha}(A)$ называются операторами порядк $a \leqslant \alpha$, с обозначением ord $a \leqslant \alpha$.

Условимся также использовать обозначение $D_{n}(A)$ для соответствующей $\mathbb{Z}_{+}$-фильтрации алгебры $D(A)$. Элементы $a \in D_{n}(A)$ называются операторами порядк $a \leqslant n$, с обозначением ord $a \leqslant n$.

ЗАмечание 1 . Определение алгебры $D(A)$ инвариантно в терминах $\mathbb{Z}$-градуировки, поскольку условие $(\gamma)$ может быть записано в виде $\left[D, A_{1}\right] \subset D($ см. также п. 1.5).

ЗАмечание 2. Элементы алгебры $D$ можно интерпретировать как диффференциальные операторы "с постоянными коэффициентами" в алгебре $A$.

ЗАмечАниЕ 3 . Определение алгебры $D(A)$ естественно обобщается на случай $\Gamma_{+}$-градуированной алгебры $A$. Более того, пусть $D(\Phi)$ - аналог алгебры $D$, получаемый заменой условия $(\beta)$ условием $D_{0}=\Phi$, где $\Phi$ - фиксированная подалгебра в $A_{0}$. Согласно $(\alpha),(\gamma)$ это определение корректно только при $\Phi \subset Z(A)$, где $Z(A)$ - центр алгебры $A$.

ЗАмечание 4 . Если $A=Z(A)_{0}$, то $D(A)$ совпадает с известной алгеброй дифференциальных операторов в коммутативной (тривиально градуированной) алгебре $A$. 
1.9. ПреДЛОЖЕНИЕ. Каждый оператор $a \in D_{-\alpha}$ однозначно определяется своими значениями на подпространстве $A_{\alpha}$. Иначе говоря, если $a=b н а$ $A_{\alpha}$ для әлементов $a, b \in D_{-\alpha}$, то $a=b$.

ДокАЗАТельство. Достаточно проверить, что равенство $a=0$ на $A_{\alpha}$ влечет $a=0$. При $\alpha=0$ это очевидно. Полагая $\alpha \neq 0$, заметим, что $\left[a, x_{i}\right]_{\varepsilon}=0$ на $A_{\alpha-\alpha_{i}}$ для всех $i \in I$, откуда $\left[a, x_{i}\right]_{\varepsilon}=0$ (допушение индукции по $|\alpha|$ ). Следовательно, $[a, x]_{\varepsilon}=0$ для всех $x \in A$. В частности, $a x=\lambda x a$ для всех однородных $x \in A$, где $\lambda=\varepsilon(a, x)$. Применяя это операторное равенство к элементу $1 \in A$ и учитывая, что $a(1)=0$ (условие $(\varepsilon)$ при $\beta=0$ ), получаем $a(x)=0$ для всех $x \in A$, т.е. $a=0$.

1.10. Приведем описание алгебры $D(A)$ в случае, когда $A$ - свободная конечнопорожденная алгебра с образуюшими $x_{i}(i \in I)$, наделенная $\mathbb{Z}$-градуировкой по правилу $\operatorname{deg} x_{i}=1(i \in I)$. Достаточно построить базисы векторных пространств $D_{-n}$, где $n \in \mathbb{Z}_{+}$.

Напомним, что $D_{0}=F$. Пространство $D_{-1}$ содержит базис из элементов $\partial_{i}$ $(i \in I)$, определяемых соотношениями

$$
\left[\partial_{i}, x_{j}\right]_{\varepsilon}=\delta_{i j}
$$

где $\delta_{i j}$ - символ Кронекера. Заметим, что операторы $\partial_{i}$ можно также определить как $\varepsilon$-дифференцирования алгебры $A$, определяемые на образующих следующим образом:

$$
\partial_{i}\left(x_{j}\right)=\delta_{i j}
$$

где $i, j \in I$.

Допустим, что искомый базис $a_{p}(p \in P)$ в пространстве $D_{-n}$ уже построен. Соотношение $\left[a, x_{j}\right]_{\varepsilon}=\delta_{i j} a_{p}$ для всех $j \in I$ наряду с условием $(\varepsilon)$, т.е. $a\left(A_{m}\right)=0$ при $m<n$, однозначно определяет оператор $a=a_{i p} \in D_{-n-1}$. Нетрудно видеть, что эти операторы образуют базис в $D_{-n-1}$.

Отсюда, полагая $c=\operatorname{card} I$, находим $\operatorname{dim} D_{-n-1}=c^{n}=\operatorname{dim} A_{n}$ для всех $n \in \mathbb{Z}_{+}$.

\section{§ 2. Билинейные формы}

2.1. Всюду в этом параграфе $A=(A, \varepsilon)$ есть стандартная алгебра (см. п. 1.5). Мы будем рассматривать следующую билинейную форму в алгебре $E(A)$ :

$$
\langle x, y\rangle=\delta_{\alpha \beta} x y(1) \text { при } x \in E(A)_{-\alpha}, \quad y \in E(A)_{\beta} .
$$

Иначе говоря, равенство (2.1) однозначно определяет форму $\langle\cdot, \cdot\rangle$ на $E(A) \times E(A)$, причем $\langle x, y\rangle=0$ при $\alpha \neq \beta,\langle x, y\rangle=x y(1) \in F$ при $\alpha=\beta$.

Билинейная форма (2.1) принимает следуюший вид на декартовом произведении $E(A) \times A$ :

$$
\langle x, y\rangle=\delta_{\alpha \beta} x(y) \text { при } x \in E(A)_{-\alpha}, y \in A_{\beta} .
$$

Заметим, что форма (2.2) невырождена слева на $D \times A$, т.е. равенство $\langle x, A\rangle=0$ для элементов $x \in D$ влечет $x=0$. Действительно, достаточно рассматривать однородные элементы $x \in D_{-\alpha}\left(\alpha \in \Gamma_{+}\right)$, для которых равенство $\left\langle x, A_{\alpha}\right\rangle=0$ влечет $x=0$ (предложение 1.9). 
Алгебра $A$ называется регулярной, если форма (2.2) невырождена справа, т.е. равенство $\langle D, y\rangle=0$ для элементов $y \in A$ влечет $y=0$. В этом случае форма (2.2) определяет двойственность векторных пространств $D, A$ (невырожденную на $D_{-\alpha} \times A_{\alpha}$, где $\left.\alpha \in \Gamma_{+}\right)$.

Соответственно алгебра $D(A)=A D=D A$ (см. п. 1.7) называется двойственной оболочкой алгебры $A$.

2.2. ПРЕДЛОЖЕНИЕ. Регулярность алгебры $А$ равносильна выполнению хотя бы одного из следующих двух условий:

$(\alpha)$ для кажсоого $\alpha \in \Gamma_{+}$и кажсдого $y \in A_{\alpha}$ из равенства $D_{-\alpha}(y)=0$ следует $y=0$

(ß) для кажсдого $y \in A$ из равенства $D_{+}(y)=0$, где $D_{+}=\bigoplus_{\alpha \in \Gamma_{+}} D_{-\alpha}$, следует $y \in F$.

Если А конечного типа, то регулярность алгебры А равносильна также следующему условию:

$(\gamma) \operatorname{dim} D_{-\alpha}=\operatorname{dim} A_{\alpha}$ для всех $\alpha \in \Gamma_{+}$.

Действительно, критерий $(\alpha)$ очевиден ввиду биортогональности семейств $D_{-\alpha}, A_{\alpha}\left(\alpha \in \Gamma_{+}\right)$. Ясно также, что $(\alpha)$ влечет $(\beta)$. Обратно, допустим, что $(\beta)$ выполняется, и докажем $(\alpha)$ индукцией по $|\alpha|$. У тверждение очевидно при $\alpha=0$. Полагая $\alpha \neq 0$, заметим, что элементы $z \in D_{-\beta}(y)$ содержатся в $A_{\alpha-\beta}$, и поэтому могут быть отличны от нуля только при $\alpha \geqslant \beta$. Полагая $\beta \neq 0$, находим в этом случае $|\alpha-\beta|<|\alpha|, D_{\beta-\alpha}(z)=0$, откуда ввиду допущения индукции следует $z=0$. Таким образом, равенство $D_{-\alpha}(y)=0$ влечет $D_{+}(y)=0$, т.е. $y \in F$. Однако $F \cap A_{\alpha}=0$ при $\alpha \neq 0$, т.е. $y=0$.

Если $A$ конечного типа, то условие $(\gamma)$ необходимо для двойственности между $D$ и $A$. С другой стороны, если $(\gamma)$ выполняется, то из левой невырожденности формы (2.2) (см. п. 2.1) следует ее невырожденность (поскольку форма (2.2) имеет ненулевой дискриминант).

ПримЕР. Каждая свободная конечнопорожденная алгебра $A$ регулярна (см. п. 1.10).

Ниже будут получены другие критерии регулярности алгебры $A$ в терминах операторов первого порядка, определенных в алгебре $A$ (см. пп. $2.4,3.4$ ).

2.3. Алгебра $A$ называется вполне дифференцируемой, если в ней определены операторы $\partial_{i}(i \in I)$, удовлетворяюшие соотношениям (1.11) с добавочным условием $\partial_{i}(1)=0$. Согласно п. 1.4, 1.5 имеем $\partial_{i} \in D_{-\alpha_{i}}$ для всех $i \in I$.

Заметим, что алгебра $A$ может быть представлена в виде $B / C$, где $B$ - свободная алгебра с образуюшими $x_{i}(i \in I)$, наделенная Г-градуировкой по правилу $\operatorname{deg} x_{i}=\alpha_{i}(i \in I), C$ - ее градуированный идеал, не содержаший элементов $1, x_{i}$ $(i \in I)$. Очевидно, алгебра $B$ вполне дифференцируема. Если $\partial_{i} C \subset C$ для всех $i \in I$, то каждый оператор $\partial_{i} \in \operatorname{End} B$ может также рассматриваться как элемент End $A$, т.е. алгебра $A$ вполне дифференцируема.

Обратно, если операторы $\partial_{i} \in \operatorname{End} A$ существуют, то из равенства (1.11) следует, что их действие совпадает с действием одноименных операторов $\partial_{i} \in$ End $B$ на каждом одночлене от образуюших $x_{i}(i \in I)$. При этом элементы $x \in C$ удовлетворяют соотношениям $\partial_{i} x=0$, где $\partial_{i} \in \operatorname{End} A$. Следовательно, $\partial_{i} C \subset C$ для элементов $\partial_{i} \in \operatorname{End} B$. 
Таким образом, алгебра $A=B / C$ вполне дифференцируема тогда и только тогда, когда $\partial_{i} C \subset C$ для элементов $\partial_{i} \in$ End $B$.

2.4. ПрЕДЛОЖЕНИЕ. Пусть алгебра А вполне дифферениируема, и пусть выполняется следующее условие:

$$
\bigcap_{i \in I} \operatorname{ker} \partial_{i}=F
$$

(т.е. әлементы $x \in F$ суть единственные решения системы уравнений $\partial_{i} x=0$, дде $\left.i \in I\right)$. Тогда алгебра А регулярна.

Действительно, равенство (2.3) влечет выполнение условия $(\beta)$ предложения 2.2 , т.е. алгебра $A$ регулярна.

2.5. Предположим, что алгебра $A$ вполне дифференцируема, и пусть $\Delta(A)-$ подалгебра с единицей в $D(A)$, порожденная элементами $x_{i}, \partial_{i}(i \in I)$. Заметим, что условие (1.11) переписывается следующим образом:

$$
\partial_{i} x_{j}-\varepsilon_{i j} x_{j} \partial_{i}=\delta_{i j}
$$

где $\varepsilon_{i j}=\varepsilon\left(\partial_{i}, x_{j}\right)$. Используя эти соотношения, находим

$$
\Delta(A)=A \Delta=\Delta A,
$$

где $\Delta$ - подалгебра с единищей, порожденная элементами $\partial_{i}(i \in I)$.

Интересно выяснить, когда $D(A)=\Delta(A)$, т.е. алгебра $D(A)$ порождается операторами порядка $\leqslant 1$. Частичный ответ на этот вопрос будет получен в п. 3.4.

Пример. Алгебра $A=(A, 1)=\mathbb{C}\left[x_{1}, \ldots, x_{n}\right]$ вполне дифференцируема. В этом случае $D(A)$ есть алгебра Вейля с образуюшими $x_{i}, \partial_{i}(i=1, \ldots, n)$.

2.6. Билинейная форма (.,.) в алгебре $A$ называется контравариантной, если

$$
\left(x_{i} f, g\right)=\left(f, \partial_{i} g\right), \quad\left(\partial_{i} f, g\right)=\left(f, x_{i} g\right)
$$

для всех $i \in I$ и всех $f, g \in A$.

Пусть $x \mapsto x^{\prime}$ - формальное сопряжение, определяемое на одночленах $x=$ $x_{i_{1}} \ldots x_{i_{n}}$ по правилу $x^{\prime}=\partial_{i_{n}} \ldots \partial_{i_{1}}$. Используя последовательно соотношения (2.6), находим

$$
(x, y)=(y, x)=\pi\left(x^{\prime} y\right) \cdot(1,1)
$$

для всех $y \in A$, где $\pi: A \rightarrow F-$ проекция, определяемая равенством (1.8). Соотношение (2.7) выполняется также для всех $x, y \in A$ при каждом (неоднозначном) продолжении операции $x \mapsto x^{\prime}$ на многочлены $x \in A$.

Из равенства (2.7) следует, что если в алгебре $A$ сушествует контравариантная форма, то она симметрична и единственна с точностью до множителя $(1,1) \in F$. Более того, градуировка алгебры $A$ ортогональна относительно этой формы. Действительно, пусть $x \in A_{\alpha}, y \in A_{\beta}$. Если $\alpha \neq \beta$, то можем считать, не ограничивая обшности, что $\alpha$ не меньше $\beta$, т.е. $\beta-\alpha \notin \Gamma_{+}$. Но тогда $x^{\prime} y=0$, т.е. $(x, y)=0$.

Ясно также, что $x^{\prime} y=\pi\left(x^{\prime} y\right) \in F$ для элементов $x, y \in A_{\alpha}\left(\alpha \in \Gamma_{+}\right)$. В результате получаем

$$
(x, y)=(y, x)=\left\langle x^{\prime}, y\right\rangle \cdot(1,1)
$$

для всех $x, y \in A$. Отсюда следует, что ненулевая форма (2.8) определена в алгебре $A$ тогда и только тогда, когда отображение $x \mapsto x^{\prime}$ однозначно определено в алгебре $A$. 
2.7. ПрЕДЛОЖЕНИЕ. Пусть алгебра А вполне дифференцируема. Тогда в ней существует единственная контравариантная форма (.,.), удовлетворяющая условию нормировки $(1,1)=1$. Более того, эта форма симметрична, m.e. $(x, y)=(y, x)$ для всех $x, y \in A$.

ДокАЗАТЕльСтво. Если $A$ - свободная алгебра с образующими $x_{i}(i \in I)$, то равенство (2.8) однозначно определяет искомую форму на базисных одночленах от образующих алгебры $A$.

В общем случае положим $A=B / C$ в обозначениях п. 2.3 , так что в алгебре $B$ искомая форма (2.8) существует. Заметим, что равенство (2.8) для однородных элементов может быть переписано следуюшим образом:

$$
(x, y)=x^{\prime}(y)=y^{\prime}(x) .
$$

Напомним (см. п. 2.3), что $\partial_{i} C \subset C$ для элементов $\partial_{i} \in$ End $B$. Поэтому $x^{\prime} C \subset C$ для всех $x \in B$. Полагая $x \in B_{\alpha}, y \in C_{\alpha}$, находим $x^{\prime}(y) \in C_{0}=0$, т.е. $\left(B_{\alpha}, C_{\alpha}\right)=0$ для всех $\alpha \in \Gamma_{+}$. В результате $(B, C)=0$, т.е. $C$ содержится в ядре формы (2.8).

Отсюда заключаем, что форма (2.8) может рассматриваться как контравариантная форма в алгебре $A=B / C$. Согласно п. 2.6 эта форма единственна при условии $(1,1)=1$.

2.8. Контравариантная форма (2.8), нормированная условием $(1,1)=1$, называется канонической билинейной формой в алгебре $A=(A, \varepsilon)$.

Алгебра $A$ называется невырожденной, если ее каноническая билинейная форма невырождена. В этом случае отображение $x \mapsto x^{\prime}$ для элементов $x \in \operatorname{End} A$ определено единственным образом как инволюция (сопряжение) относительно невырожденной формы (2.8).

Если алгебра $A$ невырождена, то равенство (2.8) определяет двойственность векторных пространств $A^{\prime}, A$ относительно формы (2.2). Тем более это верно для пары $D, A$ (поскольку $A^{\prime} \subset D$ ). Таким образом, из невырожденности алгебры $A$ следует ее регулярность.

Если алгебра $A$ невырождена, то $\Delta(A)$ есть алгебра с инволюцией. В этом случае матрища $\varepsilon_{i j}$ в соотношениях (2.4) должна быть симметричной. Действительно, применяя инволюцию к соотношениям $(2.4)$, находим $\left(\varepsilon_{i j}-\varepsilon_{j i}\right) x_{j} \partial_{i}=0$. Применяя этот оператор к элементу $x_{i}$, находим $\left(\varepsilon_{i j}-\varepsilon_{j i}\right) x_{j}=0$, т.е. $\varepsilon_{i j}=\varepsilon_{j i}$.

Пример. Если выполнено условие (2.3), то алгебра $A$ невырождена (см. доказательство теоремы 3,4 , импликация $(\gamma) \Rightarrow(\alpha))$.

2.9. ПРеДЛОЖЕНИЕ. Ядро $N$ билинейной формы (2.8) есть наибольший градуированный подмодуль $\Delta(A)$-модуля $A$, не содержащий әлемента 1 (т.е. $\left.N_{0}=0\right)$.

ДокаЗАТЕльство. Ясно, что $N$ содержится в семействе $S$ всех градуированных подмодулей $K \subset A$, для которых $K_{0}=0$. С другой стороны, семейство $S$ содержит наибольший подмодуль $M$ (определяемый как сумма всех подмодулей $K \in S)$. Таким образом, $N \subset M$.

Предположим, что $N \neq M$, и пусть $n$ - наименьшее из чисел $m \in \mathbb{Z}_{+}$, для которых $N_{m} \neq M_{m}$. Напомним, что $\operatorname{deg} \partial_{i}=-1(i \in I)$. Поэтому $\partial_{i} M_{n} \subset N$ для всех $i \in I$. Отсюда

$$
\left(M_{n}, x_{i} A\right)=\left(\partial_{i} M_{n}, A\right)=0,
$$


т.е. $M_{n}$ ортогонально $A_{m}$ при $m \neq 0$. Поскольку $n \neq 0$, имеем также $\left(M_{n}, A\right)=0$, т.е. $M_{n} \subset N$. Полученное противоречие означает, что $N=M$.

2.10. СледСТВИЕ. В условиях предложения 2.9 имеем:

(i) пространство $A / N$ есть простой обвект в категории $\Gamma_{+}-$-градуированных $\left(\mathbb{Z}_{+}\right.$-градуированных $) \Delta(A)$-модулей;

(ii) алгебра А невырождена тогда и только тогда, когда А есть простой оббект в категории $\Gamma_{+}$-градуированных $\left(\mathbb{Z}_{+}\right.$-градуированных $) \Delta(A)$-модулей.

Ниже будет показано (см. п. 3.6), что для алгебр конечного типа условие градуированности в (ii) можно отбросить (т.е. рассматривать $A$ в категории всех $\Delta(A)$-модулей).

2.11. ЗАмЕчАниЕ. Содержание пп. 2.6-2.10 имеет очевидную аналогию с теорией контрагредиентных алгебр (см., например, [6], [7]). В частности, если $A$ невырождена, то $D(A)$ есть невырожденная контрагредиентная алгебра в терминологии [7].

Заметим также, что из равенства $\Delta(A)=A \Delta$ следует $\Delta(A)=A \oplus L$, где $L-$ левый идеал алгебры $\Delta(A)$, порожденный элементами $\partial_{i}(i \in I)$. (Тот факт, что сумма прямая, следует из условий $\partial_{i}(1)=0$.) Если алгебра $A$ невырождена, то пространство $A \approx \Delta(A) / L$ совпадает с универсальным модулем Верма над алгеброй $\Delta(A)$ в терминологии [6], [7].

\section{§3. Алгебры конечного типа}

3.1. ТеОрема. Пусть А есть регулярная алгебра конечного типа, и пусть $A_{(\alpha)}=\bigoplus_{\beta \leqslant \alpha} A_{\beta}$. Тогда имеем:

(i) для каждой линейно независимой системы $\xi_{i} \in A_{(\alpha)} \quad(i=1, \ldots, n)$ и каждого набора $\eta_{i} \in A(i=1, \ldots, n)$ существует оператор $а \in D_{\alpha}(A)$ такой, что $a \xi_{i}=\eta_{i}$ для всех $i=1, \ldots, n$;

(ii) каждое из разложсений $D(A)=A D=D A$ свободно, т.е. изоморфно тензорному произведению своих компонент, относительно отображсений вида $a \otimes b \mapsto a b ;$

(iii) A есть простой $D(A)$-модуль.

ДокАЗАТЕЛЬСтво. (i) Достаточно рассматривать случай, когда элементы $\xi_{i}$ однородны. Пусть $\delta_{i}(i=1, \ldots, n)$ - дуальная система в пространстве $D_{(\alpha)}=\bigoplus_{\beta \leqslant \alpha} D_{-\beta}$ (существование этой системы следует из невырожденности формы (2.2) в конечномерных пространствах $\left.D_{-\beta} \times A_{\beta}\right)$. Таким образом, $\left\langle\delta_{i}, \xi_{j}\right\rangle=\delta_{i j}$ для всех $i, j=1, \ldots, n$. Полагая $\delta_{i} \in D_{-\beta}, \xi_{j} \in A_{\gamma}$, находим $\delta_{i}\left(\xi_{j}\right)=0$ при $\beta>\gamma, \delta_{i}\left(\xi_{j}\right)=\delta_{i j}$ при $\beta=\gamma$. Поэтому нумерация $i=1, \ldots, n$ может быть выбрана таким образом, чтобы $\delta_{i}\left(\xi_{j}\right)=\delta_{i j}$ при $i \leqslant j$.

Применяя к элементам $\delta_{i}$ процесс ортогонализации, получаем операторы $a_{i} \in D_{\alpha}(A)$, удовлетворяюшие соотношениям $a_{i}\left(\xi_{j}\right)=\delta_{i j}$ для всех $i, j=1, \ldots, n$. А именно операторы $a_{i}(i=1, \ldots, n)$ определяются рекуррентно следуюшим образом:

$$
a_{i}=\delta_{i}-\sum_{k<i} x_{i k} a_{k}
$$

где $x_{i j}=\delta_{i j}\left(\xi_{j}\right)$ при $i>j$. Заметим, что $\operatorname{deg} \delta_{i}=-\operatorname{deg} \xi_{i}$, поэтому из равенства (3.1) получаем (рекуррентно) $\operatorname{deg} a_{i}=\operatorname{deg} \delta_{i}$ для всех $i=1, \ldots, n$. Искомый 
оператор $a \in D_{\alpha}(A)$ определяется теперь следующим образом:

$$
a=\sum_{i=1}^{n} \eta_{i} a_{i}
$$

(ii) Пусть $\xi_{i}(i \in J)$ - однородный базис пространства $A$, и пусть $\delta_{i}(i \in J)$ дуальный базис пространства $D$, т.е. $\left\langle\delta_{i}, \xi_{j}\right\rangle=\delta_{i j}$ для всех $i, j \in J$. Из равенства $D(A)=A D$ следует, что $D(A)$ натянуто на элементы $\xi_{i} \delta_{j}(i, j \in J)$. Положим

$$
\sum_{i, j} c_{i j} \xi_{i} \delta_{j}=0
$$

с коэффициентами $c_{i j} \in F$, и пусть $n_{0}$ - наименьшее из чисел $n \in \mathbb{Z}_{+}$, для которых $c_{i j} \neq 0$ при некоторых $\xi_{i} \in A_{m}, \delta_{j} \in D_{-n}$. Применяя операторное равенство (3.3) к элементу $\xi_{j} \in D_{-n_{0}}$, заметим, что $\delta_{k}\left(\xi_{j}\right)=\delta_{k j}$ для всех операторов $\delta_{k}$, входящих в $(3.3)$ с ненулевыми коэффициентами. Поэтому $\sum_{i} c_{i j} \xi_{i}=0$, т.е. $c_{i j}=0$ для всех $i \in J$.

Полученное противоречие означает, что $c_{i j}=0$ для всех $i, j \in J$, т.е. элементы $\xi_{i} \delta_{j}$ образуют базис пространства $D(A)$. Таким образом, $D(A) \approx A \otimes D$, т.е. разложение $D(A)=A D$ свободно.

С другой стороны, из равенства $D(A)=D A$ следует, что $D(A)$ натянуто на элементы $\delta_{i} \xi_{j}(i, j \in J)$. Положим

$$
\sum_{i, j} c_{i j} \delta_{i} \xi_{j}=0
$$

и пусть $n_{0}$ - наибольшее из чисел $n \in \mathbb{Z}_{+}$, для которых $c_{i j} \neq 0$ при некоторых $\delta_{i} \in D_{-k}, \xi_{j} \in A_{m}, \quad k+m=n$. Применяя предложение 1.7,(i), перепишем равенство (3.4) следующим образом:

$$
\sum_{i, j} c_{i j} \xi_{j} \delta_{i}+\sum_{i, j} \sigma_{i j}=0
$$

где $\sigma_{i j}$ содержит слагаемые вида (3.3) при $k+m<n_{0}$. Отсюда по аналогии с (3.3) заключаем, что $c_{i j}=0$ при $k+m=n_{0}$. Полученное противоречие означает, что $c_{i j}=0$ для всех $i, j \in J$, т.е. разложение $D(A)=D A$ свободно.

(iii) Согласно (i) при $n=1$ модуль $A$ совпадает с каждым циклическим подмодулем $D(A) \xi_{0}$, где $0 \neq \xi_{0} \in A$. Но тогда модуль $A$ совпадает с каждым своим ненулевым подмодулем, т.е. $A$ есть простой $D(A)$-модуль.

3.2. СлЕДСТВИЕ. В условиях теоремы 3.1 имеем:

(i) алгебра $D(A)$ как векторное пространство обладает биградуировкой $A_{\alpha} D_{-\beta}$ (аналогично $\left.D_{-\beta} A_{\alpha}\right)$, где $\alpha, \beta \in \Gamma_{+}$;

(ii) каждый әлемент $a \in D_{\alpha}(A)$ записывается в виде (3.2) с коэффициентами $\eta_{i}=a\left(\xi_{i}\right)$, где $\xi_{i} \in A_{(\alpha)}$;

(iii) подпространство $A D_{-\alpha}$ совпадает с множеством всех а $\in D_{\alpha}(A)$ maких, что $а\left(A_{\beta}\right)=0$ при $\beta<\alpha$.

Действительно, (i) вытекает непосредственно из теоремы 3.1,(ii). Для доказательства (ii) достаточно использовать (3.1) в инверсной форме для элементов $\delta_{j}$, входящих в линейные комбинации типа (3.3). Наконец, для доказательства (iii) достаточно рассматривать элементы (3.2), лежащие в сумме компонент $A D_{-\beta}$ при $\beta<\alpha$. В этом случае условие $a\left(A_{\beta}\right)=0$ для всех $\beta<\alpha$ влечет $\eta_{i}=0$ для всех $i$, т.е. $a=0$. 
ЗАМЕЧАНИЕ. Согласно (i) можно дать определение строгого порядка ord $a=\alpha$ при $a \in A D_{-\alpha}$, которое согласуется с оценкой ord $a \leqslant \alpha$, определенной в п. 1.8.

3.3. ЗАмечание. Теорема 3.1 означает, что алгебра $A$ обладает "достаточно большим" запасом дифференциальных операторов. В частности, алгебра $D(A)$ плотна в End $A$ относительно поточечной сходимости в пространстве $A$.

Последнее утверждение есть частный случай известной теоремы плотности (см., например, [2]), справедливой для простых ассоциативных модулей. Теорема 3.1 , (i) содержит также уточнение этого результата для алгебры $D(A)$, относяшееся к порядкам аппроксимируюших операторов ( $\operatorname{ord} a \leqslant \alpha)$.

Другое уточнение теоремы плотности в терминах формальных рядов над алгеброй $D(A)$ будет отмечено в $\S 5$ (теорема 5.4$)$.

3.4. ТеоремА. Пусть А есть вполне дифференцируемая алгебра конечного типа (см. п. 2.3). В этом случае следующие утверждения әквивалентны:

$(\alpha)$ алгебра $А$ невырожсдена;

( $\beta$ ) алгебра А регулярна и $D(A)=\Delta(A)$;

$(\gamma)$ алгебра $A$ есть простой $\Delta(A)$-модуль;

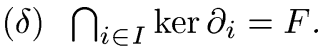

ДокАЗАТЕльство. Если $A$ невырождена, то она регулярна (см. п. 2.8), откуда $\operatorname{dim} D_{-\alpha}=\operatorname{dim} A_{\alpha}$ для всех $\alpha \in \Gamma_{+}$(см. п. 2.2). С другой стороны, $D_{-\alpha}$ содержит подпространство $\Delta_{-\alpha}=A_{\alpha}^{\prime}$, изоморфное $A_{\alpha}$, откуда $D_{-\alpha}=\Delta_{-\alpha}$ для всех $\alpha \in \Gamma_{+}$, т.е. $D=\Delta, D(A)=\Delta(A)$, что доказывает импликацию $(\alpha) \Rightarrow(\beta)$.

Импликация $(\beta) \Rightarrow(\gamma)$ вытекает непосредственно из теоремы 3.1, (iii). Заметим также, что левая часть $(\delta)$ есть градуированное подпространство в $A$. Если в этом подпространстве содержится ненулевой элемент $x_{0} \in A_{\alpha}$ при $\alpha \neq 0$, то циклический подмодуль $\Delta(A) x_{0}=A x_{0}$ не содержит 1 , что противоречит $(\gamma)$. Отсюда получаем импликацию $(\gamma) \Rightarrow(\delta)$.

Предположим теперь, что $(\delta)$ выполняется, и покажем индукцией по $n \in \mathbb{Z}_{+}$, что $\operatorname{ker} \Delta_{-n}=0$ в $A_{n}$. При $n=0$ это очевидно. Допустим, что это уже доказано при данном $n \in \mathbb{Z}_{+}$. Полагая $x \in A_{n+1}, \Delta_{-n-1}(x)=0$, находим $\Delta_{-n}\left(\Delta_{-1}(x)\right)=0$, откуда $\Delta_{-1}(x)=0$. Но тогда из условия $(\delta)$ следует $x=0$ (поскольку $F \cap$ $\left.A_{n+1}=0\right)$. Таким образом, форма (.,.) определяет двойственность векторных пространств $\Delta, A$. Согласно равенству (2.8) отсюда следует $(\alpha)$.

3.5. Замечание 1 . Равенство $D(A)=\Delta(A)$ равносильно $D=\Delta$. Действительно, записывая элемент $a \in D_{-n}$ в виде суммы элементов $a_{p} \in A_{p} \Delta_{-n-p}$ $(p \geqslant 0)$, заметим, что $a=a_{0}$ в $A_{n}$, откуда $a=a_{0}$ (предложение 1.9). Таким образом, равенство $D(A)=\Delta(A)$ влечет $D=\Delta$. Обратное очевидно.

ЗАмечание 2 . Равенство $D(A)=\Delta(A)$ в условии $(\beta)$ равносильно каждому из следующих трех условий:

(i) $D=\Delta$;

(ii) отображение $x \mapsto x^{\prime}$ инъективно в алгебре $A$;

(iii) отображение $x \mapsto x^{\prime}$ определяет инволюцию алгебры $\Delta(A)$.

Действительно, каждое из этих условий вытекает из $(\alpha)$ и влечет соотношение $D(A)=\Delta(A)$, если $A$ регулярна (импликация $(\alpha) \Rightarrow(\beta)$ в доказательстве теоремы 3.4). 
Заметим также, что равенство $D=\Delta$ влечет $D_{+}=D(A) \Delta_{-1}$ (в обозначениях п. 2.2). Поэтому импликация $(\beta) \Rightarrow(\delta)$ вытекает непосредственно из предложения 2.2 .

3.6. Теорема. Пусть А есть невырожденная алгебра конечного типа. Тогда имеем:

(i) алгебра $D(A)$ порождается әлементами $x_{i}, \partial_{i}(i \in I)$, m.е. $D(A)=$ $\Delta(A)$

(ii) алгебра $D(A)$ обладает инволюиией $x \mapsto x^{\prime}($ см. n. 2.8);

(iii) система определяющих соотношений алгебры $D(A)$ имеет следующий вид:

$$
\partial_{i} x_{j}-\varepsilon_{i j} x_{j} \partial_{i}=\delta_{i j}, \quad y_{p}=y_{p}^{\prime}=0
$$

где $i, j \in I, \quad \varepsilon_{i j}=\varepsilon_{j i}=\varepsilon\left(\partial_{i}, x_{j}\right), \quad y_{p}=0 \quad(p \in P)$ - система определяющих соотношений алгебры $A$.

ДокАЗАТЕльСтво. Утверждение (i) доказано в п. 3.4 (условие $(\beta))$. Утверждение (ii) следует из (i) и общего определения инволюции $x \mapsto x^{\prime}$ (см. п. 2.8). Докажем (iii).

Пусть $\widetilde{D}(A)$ - ассоциативная алгебра с единицей, порожденная элементами $x_{i}$, $\partial_{i}(i \in I)$ и определяюшими соотношениями (3.6). Поскольку в алгебре $D(A)$ эти соотношения выполняются, $\widetilde{D}(A)$ накрывает $D(A)$, т.е.

$$
D(A)=\widetilde{D}(A) / Z
$$

где $Z$ - градуированньй идеал алгебры $\widetilde{D}(A)$ (с градуировкой $\operatorname{deg} x_{i}=-\operatorname{deg} \partial_{i}=$ $\alpha_{i}$, определенной в $\left.\widetilde{D}(A)\right)$. Используя первую часть соотношений $(3.6)$, находим $\widetilde{D}(A)=\widetilde{A} \widetilde{D}$, где $\widetilde{A}($ соответственно $\widetilde{D})$ - подалгебра с единицей, порожденная элементами $x_{i}$ (соответственно $\left.\partial_{i}\right)$. Очевидно, $\widetilde{A}$ накрывает $A$, т.е.

$$
A=\widetilde{A} / Z \cap \widetilde{A} .
$$

В частности, $\operatorname{dim} A_{\alpha} \leqslant \operatorname{dim} \widetilde{A}_{\alpha}$ для всех $\alpha \in \Gamma_{+}$. Поскольку в алгебре $A$ выполняются определяюшие соотношения алгебры $\widetilde{A}$, имеют место также обратные неравенства, откуда $A=\widetilde{A}$ (т.е. $Z \cap \widetilde{A}=0)$. Аналогично $D=\widetilde{D}$.

Поскольку разложение $A D$ свободно в $D(A)$, оно тем более свободно в $\widetilde{D}(A)$, т.е. каждое из этих пространств изоморфно $A \otimes D$. В результате $D(A)=\widetilde{D}(A)$.

3.7. ЗАмечАниЕ. Если $\varepsilon_{i j}=1$ для всех $i, j \in I$, то алгебра $D(A)$ обладает инволюцией $x \mapsto \tilde{x}$, определяемой на образуюших по правилу $\tilde{x}_{i}=x_{i}$, $\tilde{\partial}_{i}=-\partial_{i}(i \in I)$. В этом случае разложения $D(A)=A D=D A$ сводятся друг $\mathrm{k}$ другу посредством инволюции $x \mapsto \tilde{x}$.

Остановимся подробнее на случае $\varepsilon=1$. Как видно из следующего результата, этот случай в известном смысле вырожден. Примеры невырожденных алгебр при $\varepsilon \neq 1$ будут рассмотрены в $\S 4$. 
3.8. ПРЕДЛОЖЕНИЕ. Пусть $A=(A, 1)$ - вполне дифференцируемая алгебра, u пусть $\operatorname{char} F=0$. Тогда имеем:

(i) алгебра $\Delta$ коммутативна, т.е. $\left[\partial_{i}, \partial_{j}\right]=0$ для всех $i, j \in I$;

(ii) ядро $N$ билинейной формы (.,.) есть идеал алгебры $A$, порожденный әлементами $\left[x_{i}, x_{j}\right]$, где $i, j \in I$;

(iii) факторалгебра $A / N$ изоморфна симметрической алгебре от переменHbыx $x_{i} \quad(i \in I)$.

ДокАЗАТЕЛЬСтво. (i) Полагая $\partial_{i j}=\left[\partial_{i}, \partial_{j}\right]$, находим $\left[\partial_{i j}, x_{k}\right]=0$ для всех $i, j, k \in I$, откуда $\left[\partial_{i j}, x\right]=0$ для всех $x \in A$. В частности, $\partial_{i j} x=\lambda x \partial_{i j}$ для однородных $x \in A$, где $\lambda=\varepsilon\left(\partial_{i j}, x\right)$. Отсюда $\partial_{i j}(x)=\lambda x \partial_{i j}(1)=0$, т.е. $\partial_{i j}=0$ в пространстве $A$ для всех $i, j \in I$.

(ii) Пусть $N_{0}$ - идеал алгебры $A$, порожденный элементами $x_{i j}=\left[x_{i}, x_{j}\right]$. Cогласно (i) имеем $N_{0} \subset N$. С другой стороны, фиксируем линейный порядок во множестве $I$, и пусть

$$
x(k)=\prod_{i} x_{i}^{k_{i}}
$$

(относительно данного порядка), где $k=\left(k_{i}\right) \in \mathbb{Z}_{+}^{I}-$ финитньй мультииндекс $\left(k_{i}=0\right.$, за исключением конечного числа элементов $\left.i \in I\right)$. Очевидно, одночлены (3.7) порождают базис в $A / N_{0}$. Из легко проверяемого равенства

$$
(x(k), x(r))=(1, \partial(k) x(r))=k ! \delta_{k r},
$$

где $k !=\prod_{i}\left(k_{i}\right) !, \partial(k)$ - одночлен от операторов $\partial_{i}(i \in I)$, следует, что форма (., .) невырождена в $A / N_{0}$, откуда $N_{0}=N$.

(iii) Согласно (ii) факторалгебра $A / N$ коммутативна. Более того, одночлены (3.7) образуют базис в $A / N$, т.е. $A / N$ есть симметрическая алгебра от элементов $x_{i}(i \in I)$.

3.9. СлЕДСтвиЕ. Алгебра $A=(A, 1)$ невырождена тогда и только тогда, когда она изоморфна симметрической алгебре от переменных $x_{i} \quad(i \in I)$.

\section{§4. Алгебры Серра}

4.1. Пусть $a=\left(a_{i j}\right)$, где $i, j \in I,-$ обобщенная матрица Картана [8], т.е. $a_{i j} \in \mathbb{Z}$ для всех $i, j \in I ; a_{i i}=2 ; a_{i j} \leqslant 0$ при $i \neq j ; a_{i j}=0$ влечет $a_{j i}=0$. В дальнейшем мы предполагаем, что матрица $a$ симметризуема, т.е.

$$
a_{i j}=\left\langle h_{i}, \alpha_{j}\right\rangle=\frac{2\left(\alpha_{i}, \alpha_{j}\right)}{\left(\alpha_{i}, \alpha_{i}\right)}
$$

где $h_{i} \in \mathfrak{h}, \alpha_{i} \in \mathfrak{h}^{*}, \mathfrak{h}^{*}$ - конечномерное векторное пространство над полем $\mathbb{C}$, $\langle.,\rangle-$. каноническая билинейная форма в $\mathfrak{h} \times \mathfrak{h}^{*},(.,$.$) - ассоциированное скалярное$ произведение в $\mathfrak{h}^{*}$, нормированное так, чтобы $\left(\alpha_{i}, \alpha_{i}\right) \in \mathbb{Z}$ для всех $i \in I$.

Матрица (4.1) назьвается матрицей Картана, если она положительно определена над $\mathbb{Z}$ и невырождена $(\operatorname{det} a \neq 0)$. В этом случае $a_{i j}=0,-1,-2,-3$ при $i \neq j$. Более того, скалярное произведение в $\mathfrak{h}^{*}$ можно нормировать так, чтобы $\left(\alpha_{i}, \alpha_{i}\right)=1,2,3$ для всех $i \in I$.

Матрица (4.1) называется простой (или связной), если ее невозможно представить в виде нетривиальной суммы $a^{\prime} \oplus a^{\prime \prime}$ двух матрищ вида (4.1) посредством 
разбиения множества $I$ в сумму $I^{\prime} \cup I^{\prime \prime}$. Каждая матрища (4.1) есть прямая сумма простых матриц вида (4.1) [8].

В частности, каждая простая матрица Картана относится к одному из картановских типов $A_{n}(n \geqslant 1), B_{n}(n \geqslant 2), C_{n}(n \geqslant 3), D_{n}(n \geqslant 4), E_{6}, E_{7}, E_{8}, F_{4}, G_{2}$ (см., например, [1]). Здесь нижний индекс $n$ означает card $I$ (ранг или размерность матрицы (4.1)).

ПримЕР. Все простые матрицы Картана ранга 2 относятся к одному из типов $A_{2}, B_{2}, G_{2}$.

4.2. ОПРЕДЕЛЕНИЕ. Пусть $X=X(a)$-ассоциативная алгебра с единицей над полем $\mathbb{C}$, порожденная элементами $x_{i}(i \in I)$ и определяюшими соотношениями следуюшего вида:

$$
\left(\operatorname{ad} x_{i}\right)^{1-a_{i j}} x_{j}=0 \text { при } i \neq j,
$$

где $(\operatorname{ad} x) y=[x, y]-$ коммутатор в алгебре $X$.

Соотношения (4.2) обычно называют условиями Серра [3], [14]. Соответственно будем говорить, что $X$ есть алгебра Серра, ассоциированная с матрицей (4.1).

Положим $x_{i}^{(n)}=x_{i}^{n} / n$ ! при $n \in \mathbb{Z}_{+}, x_{i}^{(n)}=0$ при $n<0$. Положим

$$
\sigma_{i j}(x)=\sum_{n}(-1)^{n} x_{i}^{(b-n)} x_{j} x_{i}^{(n)}
$$

где $b=1-a_{i j}$. Легко проверить, что левая часть соотношения (4.2) есть $b ! \sigma_{i j}(x)$.

Таким образом, условия Серра могут быть записаны в виде

$$
\sigma_{i j}(x)=0 \text { при } i \neq j .
$$

Заметим также, что алгебра $X$ изоморфна универсальной обертывающей алгебре $U(\mathfrak{n})$, где $\mathfrak{n}$ - алгебра Ли с образуюшими $x_{i}(i \in I)$ и определяюшими (лиевскими) соотношениями (4.2).

ПримеР 1. Условия Серра при $a_{i j}=0$ сводятся к условиям перестановочности элементов $x_{i}, x_{j}$.

ПримеР 2. Условия Серра для алгебры типа $A_{2}$ имеют следуюший вид:

$$
x^{2} y-2 x y x+y x^{2}=0,
$$

где $x=x_{i}, y=x_{j}$ при $i \neq j(i, j=1,2)$.

Пусть $Q$ - решетка (аддитивная подгруппа) в $\mathfrak{h}^{*}$, порожденная элементами $\alpha_{i}$ $(i \in I)$. Полагая $\operatorname{deg} x_{i}=\alpha_{i}(i \in I)$, заметим, что условия Серра (4.2) однородны относительно этой градуировки. Поэтому $X=X(a)$ есть стандартная $Q_{+}$-градуированная алгебра, где $Q_{+}=\sum_{i} \mathbb{Z}_{+} \alpha_{i}$.

4.3. Пусть $F=\mathbb{C}(q)$ - поле комплексных рациональных функций от независимой переменной $q$. Положим

$$
\begin{gathered}
q_{i}=q^{\left(\alpha_{i}, \alpha_{i}\right)} \quad \theta_{i}=q_{i}-q_{i}^{-1} \\
{[n]_{i}=\theta_{i}^{-1}\left(q_{i}^{n}-q_{i}^{-n}\right) \quad[n]_{i} !=[1]_{i} \ldots[n]_{i}}
\end{gathered}
$$


где $i \in I, n \in \mathbb{Z}_{+}$. Заметим, что $[n]_{i} \equiv n \bmod (q-1) R$, где $R=\mathbb{Z}\left[q, q^{-1}\right]$. Элементы $[n]_{i}$ называются квантовыми чельми числами (соответственно $[n]_{i} !-$ квантовый факториал).

Пусть $\tau_{i j}(x)$ - аналог многочлена (4.3), в котором $x_{i}^{(n)}=x_{i}^{n} /[n]_{i}$ ! при $n \in \mathbb{Z}_{+}$. Тогда имеем

$$
\tau_{i j}(x) \equiv \sigma_{i j}(x) \quad \bmod (q-1) R[x],
$$

где $R[x]$ - кольцо многочленов от переменных $x=\left(x_{i}\right)$ с коэффициентами из $R$.

4.4. ОПРЕДЕЛЕНИЕ. Пусть $X=X_{q}(a)$ - ассоциативная алгебра с единицей над полем $F$, порожденная элементами $x_{i}(i \in I)$ и определяюшими соотношениями следуюшего вида:

$$
\tau_{i j}(x)=0 \text { при } i \neq j .
$$

Соотношения (4.4) обычно называются квантовыми условиями Серра (см., например, [3]). Соответственно будем говорить, что $X$ есть квантовая алгебра Серра, ассоциированная с матрицей (4.1).

Полагая $\operatorname{deg} x_{i}=\alpha_{i}$ и повторяя рассуждения, приведенные в конще п. 4.2, находим, что $X=X_{q}(a)$ есть стандартная $Q_{+}$-градуированная алгебра. Ясно также, что $2(\alpha, \beta) \in \mathbb{Z}$ для всех $\alpha, \beta \in Q$ (см. п. 4.1). Поэтому функция

$$
\varepsilon_{n}(\alpha, \beta)=q^{2 n(\alpha, \beta)} \text { при } \alpha, \beta \in Q,
$$

где $n \in \mathbb{Z}$, есть мультипликатор групшы $Q$ со значениями в кольце $R=\mathbb{Z}\left[q, q^{-1}\right]$. Легко проверить, что левая часть (4.2) при $(\operatorname{ad} x) y=[x, y]_{\varepsilon}, \varepsilon=\varepsilon_{ \pm 1}$, есть $b ! \tau_{i j}(x)$. Поэтому квантовые условия Серра записываются в виде (4.2) при $\varepsilon=\varepsilon_{ \pm 1}$.

Пусть $Y_{q}(a)$ - аналог алгебры $X_{q}(a)$, получаемьй при замене поля $F$ кольцом $R$. Тогда имеем

$$
X(a)=Y_{q}(a) /(q-1) Y_{q}(a) .
$$

В этом смысле $X(a)$ есть специализация алгебры $X_{q}(a)$ (или $Y_{q}(a)$ ) в точке $q=1$.

Соответственно алгебра $X_{q}(a)$ (или $\left.Y_{q}(a)\right)$ называется квантовой деформацией алгебры $X(a)$.

4.5. ПрЕДЛОЖЕНИЕ. Алгебра $X=(X, \varepsilon)$ вполне дифференцируема при $\varepsilon=1$ в случае $X=X(a)$ и при $\varepsilon=\varepsilon_{ \pm 1}$ в случае $X=X_{q}(a)$.

ДокАЗАТЕЛЬСТво. Положим $X=B / C$ в обозначениях п. 2.3. Легко проверить следующие тождества:

$$
\begin{aligned}
{\left[\partial_{i},\left[x_{j}, f\right]\right] } & =\left[x_{j},\left[\partial_{i}, f\right]\right], \\
{\left[\left[f, x_{j}\right], \partial_{i}\right] } & =\left[\left[f, \partial_{i}\right], x_{j}\right],
\end{aligned}
$$

где $i, j \in I, f \in B$ и символ [., .] означает $\varepsilon$-коммутатор в алгебре $E(B)$. Применяя эти тождества последовательно, находим

$$
\begin{aligned}
& {\left[\partial_{i}, \tau_{j k}(x)\right]=0 \text { при } i \neq j, k,} \\
& {\left[\partial_{j}, \tau_{j k}(x)\right]=\left[\tau_{j k}(x), \partial_{k}\right]=0 .}
\end{aligned}
$$

Применяя полученные тождества к элементу $1 \in B$, находим $\partial_{i}\left(\tau_{j k}(x)\right)=0$ для всех $i, j, k \in I(j \neq k)$. Поэтому $\partial_{i} C \subset C$ для всех $i \in I$, т.е. алгебра $X$ вполне дифференцируема (см. п. 2.3). 
ЗАмечаниЕ. Легко проверить, что алгебра $(X, \varepsilon)$, где $X=X_{q}(a), \varepsilon=\varepsilon_{n}$, вполне дифференцируема только при $n= \pm 1$.

4.6. Лемма [9]. Алгебра $X_{q}($ a) невырождена.

ЗАмечАниЕ. Доказательство этой леммы, приведенное в [9] (см. также [3]), основано на теории представлений алгебры $U_{q}(\mathfrak{g})$ (см. п. 4.9). Ниже будет отмечено (см. п. 4.10) другое доказательство, пригодное для матриц Картана (4.1) и основанное на явном вычислении соотношений биортогональности в алгебре $X_{q}(a)$ (см. [4], [5]).

4.7. Теорема. Алгебра $D(X)$, әде $X=X_{q}(a)$, есть ассоциативная алгебра с единичей, порожденная әлементами $x_{i}, \partial_{i}(i \in I)$ и определяющими соотношениями следующего вида:

$$
\begin{gathered}
{\left[\partial_{i}, x_{j}\right]_{\varepsilon}=\delta_{i j},} \\
\tau_{i j}(x)=\tau_{i j}(\partial)=0 \quad \text { npu } \quad i \neq j,
\end{gathered}
$$

где $\varepsilon=\varepsilon_{ \pm 1}($ структурный коэффичиент алгебры $E(X)), \tau_{i j}(\partial)-$ результат подстановки $x_{i} \mapsto \partial_{i}(i \in I)$ в многочлен $\tau_{i j}(x)$. Алгебра $D(X)$ обладает инволючией $x \mapsto x^{\prime}$, определяемой на образующих по правилу $x_{i}^{\prime}=\partial_{i}, \partial_{i}^{\prime}=x_{i}$ $(i \in I)$.

Действительно, это утверждение есть частный случай теоремы 3.6 при $A=X$. Следует только отметить, что $\tau_{i j}(x)^{\prime}=c_{i j} \tau_{i j}(\partial)$, где $0 \neq c_{i j} \in F$. Поэтому структурные соотношения (3.6) записываются в виде (4.6), (4.7) для алгебры $D(X)$.

4.8. СледСтвиЕ. Алгебра $D(X)$ изоморфна алгебре "квантовых бозонов" $B_{q}(\mathfrak{g})$, определенной в [9] (см. также [3]) при $\varepsilon=\varepsilon_{1}$.

Таким образом, алгебра $B_{q}(\mathfrak{g})$ может быть охарактеризована как полная алгебра дифференциальных операторов в алгебре $X$ в смысле определения 1.8.

ЗАмечАние. Алгебра $X$ обладает автоморфизмом $x \mapsto \bar{x}$, определяемым по правилу $\bar{x}_{i}=x_{i}(i \in I), \bar{q}=q^{-1}$. Соответственно алгебры $D^{ \pm}(X)=D(X)$ при $\varepsilon=\varepsilon_{ \pm 1}$ сопряжены друг другу относительно индуцированного автоморфизма алгебры End $X$ :

$$
\overline{D \pm(X)}=D^{\mp}(X) .
$$

Здесь $a \mapsto \bar{a}$ - автоморфизм алгебры End $X$, определяемый по правилу $\bar{a}(\bar{x})=\overline{a x}$, где $x \in X$. Для образующих алгебры $D(X)$ этот автоморфизм определяется по правилу $\bar{x}_{i}=x_{i}, \bar{\partial}_{i}=\partial_{i}, \bar{q}=q^{-1}$.

В [9] показано также, что $X$ есть простой $B_{q}(\mathfrak{g})$-модуль. В нашем контексте этот результат есть следствие теоремы 3.1 , (iii).

В заключение этого параграфа остановимся несколько подробнее на некоторых специальных свойствах алгебр $X=X(a), X_{q}(a)$.

4.9. Пусть $\mathfrak{g}=\mathfrak{g}(a)$ - симметризуемая алгебра Каца-Муди, ассоциированная с матрицей (4.1), $A=U_{q}(\mathfrak{g})$ - ее квантовая оболочка, т.е. алгебра Дринфельда-Джимбо, ассоциированная с $\mathfrak{g}$ (см., например, [3]). Как известно, алгебра $A$ допускает следующее "треугольное разложение":

$$
A=X H Y \approx X \otimes N \otimes Y,
$$


где $H$ - коммутативная подалгебра с единищей, $X \approx Y \approx X_{q}(a)$ - квантовые алгебры Серра. При этом подалгебра $B(X)=X H=H X$ (борелевская оболочка алгебры $X)$ восстанавливается по алгебре $X$ как тензорное произведение $X \otimes H$, наделенное следующим законом умножения:

$$
h x=x h_{\alpha} \text { при } h \in H, \quad x \in X_{\alpha},
$$

где $h \mapsto h_{\alpha}\left(\alpha \in Q_{+}\right)$- автоморфизм алгебры $H$, определенный в [3]. Как показано в [3], алгебра $A$ также восстанавливается по своей компоненте Серра $X=X_{q}(a)$ как некоторая подалгебра $T(X) \subset$ End $B(X)$. А именно алгебра $T(X)$ порождается подалгеброй $B(X)$ и операторами $y_{i}(i \in I)$, порождаюшими $Y$ и определяемыми следуюшим образом: $y_{i}=a_{i} \otimes 1$ в $X \otimes H$, где

$$
a_{i}=\theta_{i}^{-1}\left(t_{i} \partial_{i}-\bar{t}_{i} \bar{\partial}_{i}\right)
$$

Здесь $t_{i}, \bar{t}_{i}$ - образующие подалгебры $H, \partial_{i}\left(\right.$ соответственно $\left.\bar{\partial}_{i}\right)$ - образующие подалгебры $D^{+}(X)$ (соответственно $\left.D^{-}(X)\right)$.

Универсальная обертывающая алгебра $U(\mathfrak{g})$ алгебры $\mathfrak{g}$ получается из $U_{q}(\mathfrak{g})$ посредством специализации $q=1, t_{i}=1$ для всех $i \in I$ (см., например, [3]). При этом алгебра $U(\mathfrak{g})$ также имеет вид (4.8), где $X \approx Y \approx X(a)$.

ЗАмЕчаниЕ. Аналогия между алгебрами $D(X), T(X)$ преврашается в асимптотическое соответствие в "кристаллическом пределе" $q=0$ [9] (см. также [3]).

4.10. В частности, пусть $а$ есть матрища Картана, $\Phi$ - система корней алгебры $\mathfrak{g}=\mathfrak{g}(a), \Phi_{+} \subset \Phi-$ подсистема положительных корней с простыми корнями $\alpha_{i}(i \in I)$. Как показано в [10] (см. также [3]), алгебра $X=X_{q}(a)$ обладает “базисом Люстига" из одночленов следуюшего вида:

$$
f(k)=f_{\alpha(1)}^{\left(k_{1}\right)} \ldots f_{\alpha(m)}^{\left(k_{m}\right)}
$$

где $k=\left(k_{1}, \ldots, k_{m}\right) \in \mathbb{Z}_{+}^{m}$, элементы $\alpha(1), \ldots, \alpha(m)$ образуют так называемый нормальный порядок системы $\Phi_{+}[3], f_{\alpha}\left(\alpha \in \Phi_{+}\right)$- обобщенные корневые векторы алгебры $X$ и символ $f_{\alpha}^{(n)}$ определяется по аналогии с частным случаем $f_{\alpha}=x_{i}$ при $\alpha=\alpha_{i}$ (см. п. 4.3).

Как показано в [5], базис Люстига (4.9) биортогонален сопряженному базису $\bar{f}(k)=\overline{f(k)}$, т.е.

$$
(\bar{f}(k), f(r))=c(k) \delta_{k r},
$$

где $0 \neq c(k) \in F$. Отсюда непосредственно вытекает невырожденность формы $(.,$.$) , т.е. лемма 4.6$ справедлива для алгебры $X_{q}(a)$.

В [4], [5] показано также, что $c(k)$ есть одночлен от элементов $\theta_{i} q_{i}^{2}(i \in I)$.

ЗАмЕчАнИЕ. В предельном случае $q=1$ форма $(.,$.$) вырождается, что соот-$ ветствует обшему правилу п. 3.10, т.е. алгебра $X=X(a)$ вырождена в смысле п. 2.8. Этот факт легко проверить непосредственно, исходя из равенства (4.10), где $c(k) \neq 0$ при $q=1$ лишь в том случае, когда $k_{i}=0$ для всех непростых $\alpha(i)$, т.е. (4.9) есть одночлен от образуюших $x_{i}(i \in I)$ алгебры $X$. 


\section{§5. Невырожденные алгебры}

5.1. Пусть $A$ - градуированная алгебра со структурным коэффициентом $\varepsilon$. Определим умножение в $A \otimes A$ по правилу

$$
(a \otimes b)(x \otimes y)=\varepsilon(b, x)^{-1}(a x \otimes b y)
$$

для однородных элементов алгебры $A$. Легко проверить, что это умножение ассоциативно. Аналогично, равенство

$$
(a \otimes b \otimes c)(x \otimes y \otimes z)=\varepsilon(b, x)^{-1} \varepsilon(c, x y)^{-1}(a x \otimes b y \otimes c z)
$$

определяет умножение в $A \otimes A \otimes A$.

В частности, пусть $A$ - вполне дифференцируемая алгебра. Каноническая билинейная форма в алгебре $A$ переносится на тензорный квадрат $A \otimes A$ по обычному правилу

$$
(a \otimes b, x \otimes y)=(a, x)(b, y),
$$

где $a, b, x, y \in A$.

5.2. Лемма. Пусть $A=(A, \varepsilon)$ - свободная алгебра (см. n. 2.3), и пусть

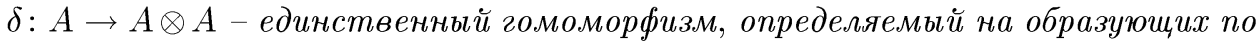
правилу

$$
\delta\left(x_{i}\right)=x_{i} \otimes 1+1 \otimes x_{i},
$$

c добавочным условием $\delta(1)=1 \otimes 1$. Тогда отображение $\delta$ сопряжено умножению в алгебре А относительно формы (5.1), т.е.

$$
(\delta(a), x \otimes y)=(a, x y)
$$

для всех $a, x, y \in A$.

Действительно, (5.3) очевидно при $a=1$. В общем случае достаточно использовать индуктивный переход $a \mapsto x_{i} a$, где $i \in I$. Заметим вначале, что форма (5.2) удовлетворяет следуюшим соотношениям:

$$
((u \otimes v) f, x \otimes y)=\varepsilon\left(v, u^{\prime} x\right)^{-1}\left(f, u^{\prime} x \otimes v^{\prime} y\right)
$$

для элементов $f \in A \otimes A$ и однородных элементов $u, v, x, y \in A$. Здесь $u \mapsto u^{\prime}-$ операция сопряжения в алгебре $A$, определяемая по правилу $x_{i} \mapsto \partial_{i}$ (см. п. 2.6). Действительно, (5.4) легко проверяется для элементов вида $f=a \otimes b$, где $a, b-$ однородные элементы алгебры $A$. Полагая $\varepsilon_{i}=\varepsilon\left(\partial_{i}, x\right)=\varepsilon\left(x_{i}, x\right)^{-1}$ и используя (5.4), находим для каждого $i \in I$

$$
\begin{aligned}
\left(\delta\left(x_{i} a\right), x \otimes y\right) & =\left(\left(x_{i} \otimes 1+1 \otimes x_{i}\right) \delta(a), x \otimes y\right)=\left(\delta(a), \partial_{i} x \otimes y+\varepsilon_{i} x \otimes \partial_{i} y\right) \\
& =\left(a, \partial_{i} x \cdot y+\varepsilon_{i} x \cdot \partial_{i} y\right)=\left(a, \partial_{i}(x y)\right)=\left(x_{i} a, x y\right)
\end{aligned}
$$

согласно индуктивному предположению (5.3). Таким образом, (5.3) доказано для всех $a, x, y \in A$. 
5.3. СлЕДСТВИЕ. Ядро $N$ канонической билинейной формы в алzебре $A$ есть идеал алгебры $A$.

Действительно, пусть $z=x y$, где $x \in N$ либо $y \in N$. Согласно (5.3) имеем

$$
(a, z)=(\delta(a), x \otimes y)=\sum_{i}\left(a_{i}, x\right)\left(a^{i}, y\right)=0
$$

для каждого $a \in A$, где положено $\delta(a)=\sum_{i} a_{i} \otimes a^{i}$. В результате $z \in N$, т.е. $N$ есть идеал алгебры $A$.

\section{4. СледСтвиЕ. Факторалгебра $A / N$ невырождена.}

Заметим также, что $A$ есть биалгебра с коумножением $\delta$, определенным в п. 5.2, и коединицей $\eta(a)=a_{0}$ (проекция на $F=A_{0}$ в алгебре $A$ ). Поскольку $\delta(N)=$ $\eta(N)=0$, все эти структуры переносятся в $A / N$.

5.5. ПреДЛОЖЕнИЕ. Алгебра A/N, определенная в $n .5 .4$, есть единственная, с точностью до изоморфизма, невырожденная алгебра с образующими $x_{i} \quad(i \in I)$ и структурным коэффициентом $\varepsilon$.

Действительно, все такие алгебры могут быть представлены, с точностью до изоморфизма, в виде $A / C$, где $C$ - идеал алгебры $A$, содержащийся в $N$ (см. п. 2.7, где свободная алгебра обозначена символом $B$ ). С другой стороны, из невырожденности алгебры $A / N$ следует $N \subset C$. Таким образом, $C=N$.

5.6. Пример. Квантовая алгебра Серра $X=X_{q}(a)$ есть единственная, с точностью до изоморфизма, невырожденная алгебра со структурным коэффициентом $\varepsilon=\varepsilon_{n}$ (см. п. 4.4), где $0 \neq n \in \mathbb{Z}$.

При $n= \pm 1$ это вытекает непосредственно из предложения 5.5. В общем случае достаточно использовать автоморфизм основного поля $\mathbb{C}(q)$, определяемьй по правилу $q \mapsto q^{n}$.

5.7. ЗАмЕчАниЕ. Квантовые условия Серра можно вывести непосредственно из условия невырожденности канонической билинейной формы в алгебре $X$.

\section{§6. Алгебра $\Pi(A)$}

6.1. Пусть $A$ - конечнопорожденная (для простоты изложения) стандартная алгебра (см. п. 1.5). Рассмотрим следующее семейство векторных пространств, ассоциированных с алгеброй $D(A)$ :

$$
P_{n}(A)=D(A) / D_{+}(n),
$$

где $n \in \mathbb{Z}_{+}, D_{+}(n)$ - сумма подпространств $D(A) D_{-k}$ при $k \geqslant n+1$.

Очевидно, семейство (6.1) возрастает с ростом $n$, т.е. $P_{n}(A)$ можно рассматривать как факторпространство пространства $P_{m}(A)$ при $n \leqslant m$. Соответственно в этом случае определена каноническая проекция $\pi_{n m}: P_{m}(A) \rightarrow P_{n}(A)$. Положим

$$
P(A)=\lim _{n} P_{n}(A)
$$


(проективный предел пространств $\left.P_{n}(A)\right)$. Элементы $a \in P(A)$ суть согласованные последовательности вида $a=\left(a_{n}\right)$, где $a_{n} \in P_{n}(A)$. Согласованность последовательности $a=\left(a_{n}\right)$ означает, что $a_{n}=\pi_{n m}\left(a_{m}\right)$ при $n \leqslant m$.

Предположим, что пространства $A D_{-n}\left(n \in \mathbb{Z}_{+}\right)$линейно независимы (например, это верно, если алгебра $A$ регулярна). В этом случае $P_{n}(A)$ естественно отождествляется с прямой суммой $A D_{0} \oplus \cdots \oplus A D_{-n}$, так что

$$
P_{n}(A)=P_{n-1}(A) \oplus A D_{-n},
$$

где $P_{-1}(A)=0$. Соответственно $P(A)$ можно рассматривать как пространство формальных рядов вида $a=\sum_{n} \delta a_{n}$ с компонентами $\delta a_{n}=a_{n}-a_{n-1}$ (так что $\left.\delta a_{0}=a_{0}\right)$.

В частности, пусть $A D \approx A \otimes D$ (что верно, если $A$ регулярна). В этом случае мы будем записьвать элементы $a \in P(A)$ в виде следуюших формальных рядов:

$$
a=\sum_{i, j} a_{i j} \xi_{i} \delta_{j}
$$

где $\xi_{i} \in A, \delta_{i} \in D(i=0,1, \ldots)$ - однородные базисы, $\xi_{0}=\delta_{0}=1$, с коэффициентами $a_{i j} \in F$. Заметим, что $a_{0}=\pi(a)$, где $\pi$ - проекция $P(A)$ на $F$ параллельно сумме подпространств $A_{m} D_{-n}$ при $m+n \neq 0$.

Элементы $a \in P(A)$ допускают естественную интерпретацию как линейные операторы в пространстве $A$. Действительно, из равенства $D_{+}(n) A_{(n)}=0$ следует, что каждому элементу $a_{n} \in P_{n}(A)$ можно сопоставить одноименный оператор $a_{n}: A_{(n)} \rightarrow A$, причем $a_{n}=a_{m}$ на $A_{(n)}$ при $n \leqslant m$. Поэтому равенство $a=a_{n}$ на $A_{(n)}$ однозначно определяет линейный оператор $a$ во всем пространстве $A$.

Если $A$ регулярна, то при помощи 3.2 ,(ii) находим, что отображение $P(A) \rightarrow$ End $A$ инъективно, т.е. $P(A)$ вкладывается в End $A$.

Элемент $a=\left(a_{n}\right) \in P_{n}(A)$ называется однородным степени $\alpha \in \Gamma$, если $\operatorname{deg} a_{n}=\alpha$ для всех $n \in \mathbb{Z}_{+}$. Положим

$$
\Pi(A)=\bigoplus_{\alpha \in \Gamma} \Pi(A)_{\alpha},
$$

где П $(A)_{\alpha}$ - подпространство в $P(A)$, состоящее из формальных рядов степени $\alpha$.

Заметим, что П $(A)$ есть также сумма подпространств $\Pi(A)_{n}$, где $n \in \mathbb{Z}_{+}$. Элементы $a \in \Pi(A)$ суть формальные ряды (5.4) с дополнительным условием $\left|n_{i}-m_{j}\right| \leqslant$ const, где $n_{i}=\operatorname{deg} \xi_{i}, m_{i}=-\operatorname{deg} \delta_{i}\left(\right.$ так что $\left.n_{i}, m_{i} \in \mathbb{Z}_{+}\right)$.

6.2. ПРЕДЛОЖЕНИЕ. Пусть $A D \approx A \otimes D$, и пусть $\pi_{n}$ - каноническая проекиия $P(A)$ на $P_{n}(A)$, где $n \in \mathbb{Z}_{+}$. Тогда имеем:

(i) для каждой пары әлементов $a=\left(a_{n}\right), \quad b=\left(b_{n}\right) \in \Pi(A)$ последовательность $\pi_{n}\left(a_{k} b_{l}\right)$ стационарна при $k, l \rightarrow \infty$, m.e. не зависит от $k, l$ при $k \geqslant k_{0}$, $l \geqslant l_{0}$

(ii) последовательность $c_{n}=\lim _{k, l} \pi_{n}\left(a_{k} b_{l}\right)$ согласована, т.е. определяет элемент $c=a b \in \Pi(A)$. 
ДокАЗАТЕЛЬСТво. Достаточно рассматривать $\mathbb{Z}$-однородные элементы $a, b \in \Pi(A)$, для которых $\operatorname{deg} a=p, \operatorname{deg} b=q(p, q \in \mathbb{Z})$. Соответствуюшие элементы $\delta a_{k} \in A D_{-k}, \delta b_{l} \in A D_{-l}$ содержатся в компонентах $A_{s} D_{-k}, A_{r} D_{-l}$ соответственно при $s-k=p, r-l=q$. Приводя произведение этих элементов к стандартному виду $A D$ (путем перестановки элементов, входящих в $D_{-k} A_{r}$ ), находим, что это произведение может внести ненулевой вклад в $A_{m} D_{-n}$ только при $s \leqslant m, l \leqslant n$. Отсюда $k \leqslant m+p, r \leqslant n+q$. Таким образом, лишь конечное число произведений $\delta a_{k} \cdot \delta b_{l}$ может внести ненулевой вклад в $A D_{-n}$, т.е. последовательность $\pi_{n}\left(a_{k} b_{l}\right)$ стационарна. Отсюда следует также согласованность последовательности $c_{n}$, определенной в (ii).

6.3. СлЕДСТВИЕ. Векторное пространство П $(A)$ есть Г-градуированная (Z्Z-градуированная) алгебра относительно умножения формальных рядов (6.4).

Элементы алгебры П $(A)$ мы называем псевдодифференциальными операторами в алгебре $A$.

ЗАмЕчАниЕ. Определение алгебры П $(A)$ легко переносится на алгебры конечного типа, если в определении (6.2) рассматривать пределы по направленности, определяемой элементами $\alpha \in \Gamma_{+}$. Более того, условие конечности типа можно отбросить, если рассматривать ряды (6.4), имеюшие лишь конечное число ненулевых слагаемых, выделяемых условиями однородности $\operatorname{deg} \delta_{j}=$ const (см. аналогичную конструкцию для контрагредиентных алгебр, изложенную в [7]).

6.4. Теорема. Пусть А есть конечнопорожденная невырожденная алгебра. Тогда имеем $\Pi(A)=E(A)$. Иначе говоря, каждый градуированный оператор $a \in$ End $A$ есть псевдодифференциальный оператор в алгебре $A$.

ДокАЗАТЕльСтво. Пусть $E^{n}(A)$ - множество всех элементов $a \in E(A)$, удовлетворяющих условиями $a\left(A_{m}\right)=0$ при $m \leqslant n$. Согласно теореме 3.1 , (i) для каждого $a \in E^{n-1}(A)$ существует оператор $b \in D_{n}(A)$ такой, что $a-b \in E^{n}(A)$. Coгласно следствию 3.2 имеем также $b \in A D_{-n}$. Предположим теперь, что $a \in E(A)$. Применяя последовательно изложенную выше конструкцию, находим

$$
a=\delta a_{0}+\cdots+\delta a_{n}+a_{n}
$$

где $\delta a_{i} \in A D_{-i}, a_{n} \in E^{n}(A)$. Отсюда заключаем, что $a=\sum_{n} \delta a_{n} \in P(A)$. Ясно также, что конструкция ряда (6.6) сохраняет степень однородности, т.е. $a \in \Pi(A)$.

6.5. СледСтвиЕ. В условиях теоремы 6.4 каждый оператор $а \in E(A)$ записывается в виде

$$
a=\sum a_{i_{1} \ldots i_{n}} \delta_{i_{1}} \ldots \delta_{i_{n}}
$$

с коэффициентами $a_{i_{1} \ldots i_{n}} \in A$ (әде сумма берется по всем конечным наборам индексов $\left.i_{1}, \ldots, i_{n}\right)$.

При этом условие $\mathbb{Z}$-градуированности оператора (6.7) означает, что $\left|\operatorname{deg} a_{i_{1} \ldots i_{n}}-n\right| \leqslant$ const для всех $n \in \mathbb{Z}_{+}$(с константой, зависящей от оператоpa $a)$. 
6.6. ПримеР. Пусть $A=(A, 1)=\mathbb{C}[x]$, т.е. $D(A)$ есть алгебра Вейля с образуюшими $x, \delta$ и определяюшим соотношением $[\delta, x]=1$. В этом случае элементы (6.7) принимают следующий вид:

$$
a=\sum_{m, n} c_{m n} x^{m} \delta^{n}
$$

с коэффициентами $c_{m n} \in \mathbb{C}$, где $|m-n| \leqslant$ const для всех $m, n \in \mathbb{Z}_{+}$.

6.7. ЗАмечание. Результаты этой статьи легко обобщаются на $\Gamma_{+}$-градуированные алгебры с центральными компонентами $A_{0}$ (т.е. $\left.A_{0} \subset Z(A)\right)$. В действительности можно рассматривать также более обшие алгебры картановского типа [7] с "почти центральными" компонентами $A_{0}$.

Возможны также другие обобшения алгебры $D(A)$, связанные с рассмотрением более сложных коммутационных соотношений в алгебре End $A$. Примеры такого рода встречаются в теории квантовых групп (см., например, [12]).

\section{Список литературы}

1. Бурбаки Н. Группы и алгебры Ли. Гл. IV-VI. М.: Мир, 1982.

2. Диксмье Ж. Универсальные обертывающие алгебры. М.: Мир, 1977.

3. ЖКелобенко Д. П. Представления редуктивных алгебр Ли. М.: Наука, 1994.

4. Желобенко Д. П. Алгебра квантовых бозонов, фильтрация Шуберта и базисы Люстига // Изв. РАН. Сер. матем. 1993. Т. 57. №6. С. 3-32.

5. Желобенко Д. П. Фильтрация Шуберта и квазидифференцирования в квантовых алгебpax Серра // Вестн. РУДН. 1995. Т. 2. № 2. С. 3-11.

6. Zhelobenko D. P. Contragredient algebras // J. on Group Theory in Physics. 1993. V. 1. № 1. Р. 201-233.

7. Желобенко Д. П. Алгебры картановского типа // Докл. РАН. 1994. Т. 339. № 4. C. $442-445$.

8. Кач, В. Г. Бесконечномерные алгебры Ли. М.: Мир, 1993.

9. Kashiwara $M$. Crystalizing the $q$-analogue of universal enveloping algebras // Comm. Math. Phys. 1990. V. 122. P. 249-260.

10. Lusztig G. Canonical bases arizing from quantized enveloping algebras // J. of Amer. Math. Soc. 1990. V. 3. P. 447-498.

11. Manin Yu.I. Quantum groups and non-commutative geometry. Montreal: CRM Univ., 1988.

12. Noumi M., Umeda I., Wakayama $M$. A qauntum analogue of the Capelly identity and an elementary differential calculus on $\mathrm{GL}_{q}(n)$. Preprint / Univ. of Tokyo, 1991.

13. Онищик А. Л. Топология транзитивных групп преобразований. М.: Наука, 1995.

14. Серр Ж.-П. Группы и алгебры Ли. М.: Мир, 1969. 